\title{
Metachronous double malignancy involving the kidney and the breast: A case report
}

\author{
Osama Ahmed $^{1 *}$, Tahir Abbas ${ }^{1,2}$ \\ ${ }^{1}$ Department of Medicine, University of Saskatchewan, Saskatoon, Canada; *Corresponding Author: osa362@mail.usask.ca \\ ${ }^{2}$ Saskatchewan Cancer Agency, Saskatoon Cancer Centre, Saskatoon, Canada
}

Received 8 December 2013; revised 5 January 2014; accepted 3 February 2014

Copyright (C) 2014 Osama Ahmed, Tahir Abbas. This is an open access article distributed under the Creative Commons Attribution License, which permits unrestricted use, distribution, and reproduction in any medium, provided the original work is properly cited. In accordance of the Creative Commons Attribution License all Copyrights (C) 2014 are reserved for SCIRP and the owner of the intellectual property Osama Ahmed, Tahir Abbas. All Copyright (C 2014 are guarded by law and by SCIRP as a guardian.

\section{ABSTRACT}

The occurrence of multiple primary cancers is rare; it can be missed as a disease progression. The etiology remains controversial. We report a case of a 55-year-old female with metastatic renal cell carcinoma treated with sutent followed by left sided nephrectomy. Follow-up CT showed increase in the size of the right axillary lymph nodes which was proven after biopsy to be metastatic adenocarcinoma of the breast. Any suspicious disease progression in a single site not compatible with disease history should be biopsied for confirmation. The relationship between renal cell carcinoma and breast cancer is still unclear, and more case reports are required to determine this relationship.

\section{KEYWORDS}

\section{Double Malignancy; Cancer Breast; Renal Cell Carcinoma}

\section{INTRODUCTION}

The occurrence of another malignancy in a patient with a known malignant tumor is known as double malignancy [1]. It is classified into two categories depending on the time of diagnosis of each primary site. Synchronous cancers occur at the same time or within an interval of two months, while metachronous cancers follow in sequence and more than two months apart [2]. The incidence has increased in recent years possibly secondary to improve survival in cancer patients [3].

It's always a challenge to diagnose and manage double malignancy as the second tumor is always suspected to be a disease progression of the primary tumor besides the challenge of choosing a regimen that would affect both tumors.

We report a case of metachronous double malignancy involving the kidney and the breast.

\section{CASE PRESENTATION}

A 56-year-old woman was first seen in Saskatoon cancer Centre on Nov 09, 2010. She was followed by her family physician for persistent iron deficiency anemia. In August 2010 her $\mathrm{Hb}$ was $82 \mathrm{~g} / \mathrm{L}$ and abdominal ultrasound showed a mass in the left kidney. CT scan on Sep 24, 2010 showed an enhancing mass measuring approximately $8.6 \times 8.5 \times 10.5 \mathrm{~cm}$ arising from the inter-lower pole aspect of the left kidney consistent with a large renal cell carcinoma.

In addition there was significant lymphadenopathy at the level of the left renal hilum. Multiple bilateral lung nodules and masses were identified. FNA cytology and CT guided biopsy of the left renal mass showed: renal cell carcinoma, clear cell type.

Patient was diagnosed as Stage IV renal cell carcinoma and was started on Sutinib $50 \mathrm{mg}$ a day for four weeks every six weeks. During her second cycle of treatment she became significantly short of breath and was admitted to hospital where she was found to be anemic with $\mathrm{Hb} 69 \mathrm{~g} / \mathrm{L}$. Her treatment course was interrupted due to recurrent melena and low $\mathrm{Hb}$ and she needed frequent blood transfusions. After two failed colonoscopies and normal barium enema video capsule endoscopy was performed on Apr 18, 2011 which showed evidence of small bowel angiodysplasia, and she had cauterization in July 2011. Starting from cycle four Sutinib dose was reduced to $37.5 \mathrm{mg}$ for four weeks every six weeks due to persistent anemia and in Aug, 2011 same dose was given for two weeks every three weeks. 
Fortunately, follow up CT scan on Sep 23, 2011 showed a dramatic positive response with complete resolution of lung metastasis and decrease size of left renal mass to $4 \mathrm{~cm}$. Case was discussed with the urologist and patient underwent laparoscopic left nephrectomy in November 2011. Pathology showed: clear cell carcinoma with treatment effect ypT1A and ypNX. Patient was off treatment after surgery. CT on Jan 5, 2012 showed development of right axillary and mediastinal lymphadenopathy as well as early lung parenchymal metastatic disease within the left upper lobe. As this was considered a recurrence of metastatic renal cell carcinoma, Sutinib was reinitiated using $37.5 \mathrm{mg}$ daily for two weeks every three weeks. Her treatment course was complicated with Shingles, diarrhea, elevated blood pressure, and hypothyroidism. CT done on June 20, 2012 showed that right axillary lymph node has increased in size otherwise all findings were stable.

At this point, Patient continued on Sutent. Bilateral mammogram was done on Sep 20, 2012 and there was no evidence of a primary breast malignancy. Again, CT on Oct 3, 2012 showed increase in size of the right axillary lymph nodes. As the patient was still on Sutent and she had a good response but the axillary lymph nodes were increasing it was requested from the surgeon to do right axillary lymph node biopsy to rule out a second primary. Pathlogy showed evidence of metastatic adenocarcinoma suggestive of a primary breast carcinoma.

Case was discussed in breast tumor rounds and the decision was to do a mastectomy followed by adjuvant chemotherapy and possible radiotherapy. In November 26, 2012 patient had right modified radical mastectomy with 21/30 positive lymph nodes. There were two foci of invasive ductal carcinoma. Tumor was Grade 3 with positive lymphovascular invasion, negative margins, pT1b, pN3a and triple negative.

Adjuvant chemotherapy was started on Jan 15, 2013 with cycle 1 FEC100 (5-flurouracil, epirubicin, cyclophosphamide). After cycle 2, patient was admitted to hospital with neutropenic fever and prophylactic neulasta and ciprofloxacin were added to her protocol. She finished three cycles and the first cycle of docetaxel with $10 \%$ dose reduction was started on Mar 28, 2013 which was complicated by neutropenic fever which required hospital admission and IV antibiotics despite using neulasta and ciprofloxacin prophylaxis and dose reduction.

After discussion with the patient regarding benefits versus risk it was decided to hold chemo and refer the patient to Radiation oncology. It was recommended by the radiation oncologist to have radiation therapy to right chest wall, right axilla and right supraclavicular regions and patient agreed to the plan. Treatment was commenced on May 1, 2013 and completed on June 6, 2013. A dose of 5000 cGy in 25 fractions over 37 days was given. The course of treatment was tolerated generally well. Patient is currently under follow-up.

\section{DISCUSSION}

In 8667 patients treated for renal cell carcinoma in the Swedish family database, 677 had a second primary malignancy, there were 44 breast cancers. Raissouni et al. reported a case with multiple neoplasm: esophagus, breast and renal cell carcinoma [4]. There is no clear theory yet behind double malignancy etiology.

Few Theories have been suggested. First, it is quite reasonable to assume that when different tissues in same individual are exposed to same carcinogenic agent, several areas of malignant change may develop, for example smoking can affect the lungs, nasopharynx and bladder [1].

Second, there may be a genetic predisposition resulting in the cancer family syndrome. The BRCA (Breast Cancer) gene mutation would be one such example. Third, the development of a second primary cancer after treatment of the first with radiotherapy or chemotherapy is well documented [5]. This is often seen with treated hematological malignancies on childhood.

The first two scenarios could be explain our case as both cancers started either at the same time but the breast cancer was diagnosed late or the breast cancer followed the renal cancer by only few months. Our patient had not received chemotherapy or radiotherapy so therapy-related malignancy is not applicable.

Renal cell carcinoma (RCC) accounts for approximately $2 \%$ of all malignancies globally and $80 \%-85 \%$ of all malignant kidney tumors. Renal cell carcinoma is one of the most aggressive urological cancers. Up to $25 \%$ $30 \%$ of RCC patients have overt metastatic disease at initial presentation [6].

Sunitinib is an oral tyrosine kinase inhibitor that blocks several pathways central to angiogenesis including vascular endothelial growth factor receptors (VEGF) and platelet-derived growth factor receptors (PDGFR) [7].

Advances in the understanding of the pathogenesis and molecular biology of RCC have identified the importance of angiogenesis in the development of the disease. VEGF (Vascular Endothelial Growth Factor)-targeted therapies - sunitinib, sorafenib and bevacizumab-and their clinical superiority to traditional cytokine therapy in metastatic RCC has resulted in a paradigm shift in the treatment of this disease, and that was clear in the case presented as she had a complete resolution of lung nodules after treatment with sutent [6].

Angiogenesis has been a long-standing therapeutic target in malignant tumors, an idea pioneered by Judah Folkman. Preclinical studies have shown that inhibition of the vascular endothelial growth factor (VEGF) path- 
way impedes tumor growth.

The administration of antiangiogenic agents have been shown to generate intramural hypoxia which might accelerate tumor growth and metastasis by increasing Breast cancer stem cells (CSC) population [8], and this could explain axillary lymph node progression during sutent treatment. Improving efficacy of antiangiogenic agent will require the concurrent use of CSC-targeting agents.

In our discussed case the first challenge was to manage the anemia which was initially contributed to treatment but as it did persist after Sutent dose reduction, it needed further investigations as capsule endoscopy and it turned out to be angiodysplasia. Looking for different possible causes of an oncology patient presentation other than blaming the cancer or the treatment is warranted in many cases.

The second challenge was to explain persistent axillary lymphadenopathy in contrast to other stable findings. The decision to have another biopsy in a known cancer patient in a suspected lesion is reasonable especially in such a case where you had an initial good response to treatment.

\section{CONCLUSIONS}

In cancer patients, any suspicious lesion not responding to treatment, in contrast to other areas of the disease, should be biopsied as the presence of a different pathology and double malignancy is always possible.

Persistent anemia in cancer patients under treatment not responding to dose modifications should be further investigated as we might find a reversible underlying cause.

Further investigations should be conducted to identify effect of anti-angiogenesis on cancer stem cells.

\section{CONSENT}

Written informed consent was obtained from the patient for publica- tion of this case report and accompanying images. A copy of the written consent is available for review by the Editor-in-Chief of this journal.

\section{REFERENCES}

[1] Dalal, S., et al. (2008) Synchronous double malignancy: Adeno-carcinoma of caecum and renal cell carcinoma. The Internet Journal of Gastroenterology, 6, 4.

[2] Mesmoudi, M., et al. (2011) Triple malignancy in a single patient including a cervical carcinoma, a basal cell carcinoma of the skin and a neuroendocrine carcinoma from an unknown primary site: A case report and review of the literature. Journal of Medical Case Reports, 5, 462. http://dx.doi.org/10.1186/1752-1947-5-462

[3] Cheng, J.H., et al. (2008) Double primary bronchogenic carcinoma of the lung and papillary thyroid carcinoma: A case report. Journal of Medical Case Reports, 2, 309. http://dx.doi.org/10.1186/1752-1947-2-309

[4] Raissouni, S., et al. (2012) Radiation induced esophageal adenocarcinoma in a woman previously treated for breast cancer and renal cell carcinoma. BMC Research Notes, 5, 426. http://dx.doi.org/10.1186/1756-0500-5-426

[5] Fletcher, H., et al. (2007) Multiple metachronous malignancies, one patient with three primary malignancies: A case report. Journal of Medical Case Reports, 1, 15. http://dx.doi.org/10.1186/1752-1947-1-15

[6] Albiges, L. and Escudier, B. (2011) Vascular endothelial growth factor-targeted therapy for the treatment of renal cell carcinoma. Drugs, 71, 1179. http://dx.doi.org/10.2165/11591410-000000000-00000

[7] Aapro, M. (2009) Sunitinib and metastatic breast cancer. Advances in Oncology, 4, 18.

[8] Conley, S.J., et al. (2012) Antiangiogenic agents increase breast cancer stem cells via the generation of tumor hypoxia. Proceedings of the National Academy of Sciences of USA, 109, 2784-2789.

\section{LIST OF ABBREVIATIONS}

CT: Computed Tomography;

Hb: Hemoglobin;

FNA: Fine Needle Aspiration;

FEC100: 5-Flurouracil, Epirubicin, Cyclophosphamide;

BRCA: Breast Cancer;

RCC: Renal Cell Carcinoma;

VEGF: Vascular Endothelial Growth Factor Receptors;

PDGFR: Platelet-Derived Growth Factor Receptors; CSC: Cancer Stem Cells. 